\title{
Do gender and age influence agroforestry farmers' knowledge of tree species uses in an area of the Atlantic Forest, Brazil?
}

\author{
Naiana Pereira Lunellił ${ }^{\star}$, Marcelo Alves Ramos ${ }^{2}$ and Clovis José Fernandes de Oliveira Júnior ${ }^{3}$
}

Received: June 29, 2016

Accepted: November 16, 2016

\begin{abstract}
This study aimed to explore the extent of knowledge of tree species and their uses by agroforestry farmers in the Vale do Ribeira, Brazil, to verify whether gender and age are related to species knowledge in different use categories. The study was conducted using semi-structured interviews, guided tours, direct observation and collection and identification of botanical material. Analyses were based on the frequency with which plants were mentioned. Forty farmers ( 17 women and 23 men aged 18 to 78 years) were interviewed. There were significant differences in the species richness cited exclusively by each gender; the number of species mentioned by men (36 sp.) was higher than by women (11 sp.). Men exhibited a greater knowledge of such categories as construction, organic matter, fuel, animal feed, technology and beekeeping, whereas there were no significant differences for food, medical and other categories. Age was also a distinguishing factor; younger people mentioned the lowest number of species (74 sp.) compared to adults (163 sp.) and the elderly (150 sp.). The greater number of species mentioned exclusively by men may indicate the existence of specialized knowledge, whereas environmental constraints and the availability of plant resources may limit the knowledge of young people.
\end{abstract}

Keywords: agroforestry farmers, Atlantic Forest, Cooperafloresta, ethnobotany, intracultural variations, Vale do Ribeira

\section{Introduction}

Globally, several societies have the potential to offer low-cost technological solutions that are adapted to regional conditions. These societies depend on the qualitative and quantitative aspects of natural resources to survive (Kang \& Akinnifesi 2000). Studies that address rural populations are important because they can contribute to the protection and management of plant resources (Albuquerque 1997).
In particular, farmers are surrounded by a wide variety of both wild and cultivated plants (Scherr 1991). In addition, the way these populations use, manage and technologically process many of the plants may have been influenced by indigenous knowledge (Oliveira Jr \& Cabreira 2012). In this regard, rural communities hold broad knowledge of rain forest uses and can develop well-managed, complex and successful agroforests (Kang \& Akinnifesi 2000).

Agroforestry is a practice that prioritizes the use of local resources and knowledge to increase biodiversity and

\footnotetext{
${ }^{1}$ Programa de Pós-Graduação em Biodiversidade Vegetal e Meio Ambiente do Instituto de Botânica, Av. Miguel Stéfano 3687, 04301-902, São Paulo, SP, Brazil

${ }^{2}$ Departamento de Ciências Biológicas, Universidade de Pernambuco, Campus Mata Norte, Rua Prof. Amaro Maltez 201, 55800-000, Nazaré da Mata, PE, Brazil

${ }^{3}$ Núcleo de Pesquisa em Plantas Ornamentais, Instituto de Botânica, Av. Miguel Stéfano 3687, 04301-902, São Paulo, SP, Brazil

${ }^{*}$ Corresponding author: nai.lunelli@gmail.com
} 
achieve ecological balance in forest succession (Altieri \& Nicholls 2012; Lunelli et al. 2013). Trees are not only an important resource for human populations (Assogbadjo et al. 2012; Brandt et al. 2013) but also represent the main structural component of forest areas and are responsible for ecological functions that influence both biotic and abiotic environments (García-Barrios \& Ong 2004).

Local knowledge dynamics may be influenced by several factors. Literature highlights gender roles (Guimbo et al. 2011) and age (Mathez-Stiefel et al. 2012), as factors that directly influence the way people interact with plants. Social and cultural contexts determine the roles suitable for women and men and encourage them to develop different activities (Pfeiffer \& Butz 2005; Howard \& Nabanoga 2007) that may vary over time and differ by region.

Regarding gender, women are usually observed to be in charge of affairs that permeate the home environmentparticularly with regard to caring for children and other family members (Howard 2003). However, in rural areas, women are also the largest food producers, accounting for $80 \%$ of the production in Africa, $60 \%$ in Asia and $40 \%$ in Latin America (Howard 2003). Many of these women are involved in the selection and breeding of plant varieties and are genuine guardians of biodiversity (Shiva 2002). In contrast, men tend to focus their activities on paid work such as commercial crops and woodcutting activitiesmainly for construction purposes-leading them to work in locations distant from their homes (Camou-Guerrero et al. 2008; Lyon \& Hardesty 2014; Ramos et al. 2015)

Age is also a factor to consider because knowledge is traditionally transmitted from generation to generation and can be transformed over time. There is a tendency for older people to have greater knowledge of resources because experiences naturally provide greater opportunities for learning throughout life (Case et al. 2005; Lyon \& Hardesty 2014). In this context, migration (Reyes-García et al. 2005; Ceuterick et al. 2008; Gandolfo \& Hanazaki 2011), modernization (Quinlan \& Quinlan 2007) and access to plant resources (Zuchiwschi et al. 2010; Albuquerque et al. 2011) can influence knowledge variations among different age groups.

A better understanding of people's knowledge can strengthen planning efficiency when choosing species for inclusion in the Agroforest Systems (SAF), improve social acceptability and add value to the local identity. Although studies have been conducted on this topic, no welldefined pattern has emerged (Avilez et al. 2016); therefore, studies that contribute to the understanding of the role of gender knowledge of plants are needed. Based on these considerations, this study aimed to analyse the knowledge and use of tree species by agroforestry farmers in the Vale do Ribeira, Brazil, to investigate the relationships between age and gender and knowledge of tree species in different use categories.

\section{Materials and methods}

\author{
Study area
}

This study was conducted in the Vale do Ribeira region, which is located between the southeastern portion of São Paulo State (SP) and the northeastern portion of Paraná State (PR), Brazil (Fig. 1). The climate, according to the Köppen classification, is Cfa-type humid subtropical, with no dry season and a hot summer in which average temperature is approximately $23^{\circ} \mathrm{C}$ (Setzer 1966). The average annual rainfall exceeds $1,500 \mathrm{~mm}$; the highest incidence occurs in January and the lowest in August (Lepsh et al. 1990). The region has irregular relief with large altimetric gaps ranging from $2 \mathrm{~m}$ in the coastal lowlands to $1,300 \mathrm{~m}$ in the mountain zone (Alves 2004). The predominant soils are Argisol and Latosol (Red-Yellow) followed by Cambisol and Entisol (Lepsh 2002). This territory is home to the largest contiguous area of the Atlantic Forest in the country, characterized by dense rain forest (Ivanauskas et al. 2012). Currently, banana crops, cattle and buffalo farming are the most important economic activities in the region. Family farmers and settlements represent approximately $76 \%$ of the rural enterprises, which own $18 \%$ of the total agricultural area (SIT 2013). From social aspects, the population in the Vale do Ribeira has a low income and educational level, a high incidence of infant mortality and illiteracy and a lack of infrastructure and basic sanitation (Adams et al. 2013).

The Association of Agroforestry Farmers of Barra do Turvo and Adrianópolis (Cooperafloresta) started in 1996 after its founders became interested in a newly introduced production model. Historically, the slash-and-burn technique was conducted in this area prior to planting corn, beans and bananas (Neves et al. 2012). However, to meet legal requirements, even the brief time that the land was required to rest (Gomes et al. 2013) contributed to soil degradation processes that hampered agricultural productivity. Famers individually selling their crops was also a limiting factor because of the high costs involved in shipping produce to distant markets. Based on this scenario and thanks to the influence of external actors and institutions, it was possible to transform the conditions of local life.

Today, 112 families are members of the association, including a total of 322 people living in rural districts in the municipalities of Barra do Turvo (SP), Adrianópolis (PR) and Bocaiuva do Sul (PR) along the border of São Paulo and Paraná (Fig. 1). Approximately $5 \%$ of the community's population is involved in association activities. The association focuses on implementing agroecologybased agroforestry methods to generate income, recover local knowledge and educate farmers about environmental issues. This practice has fostered increased production and conservation of biological diversity and natural resources 

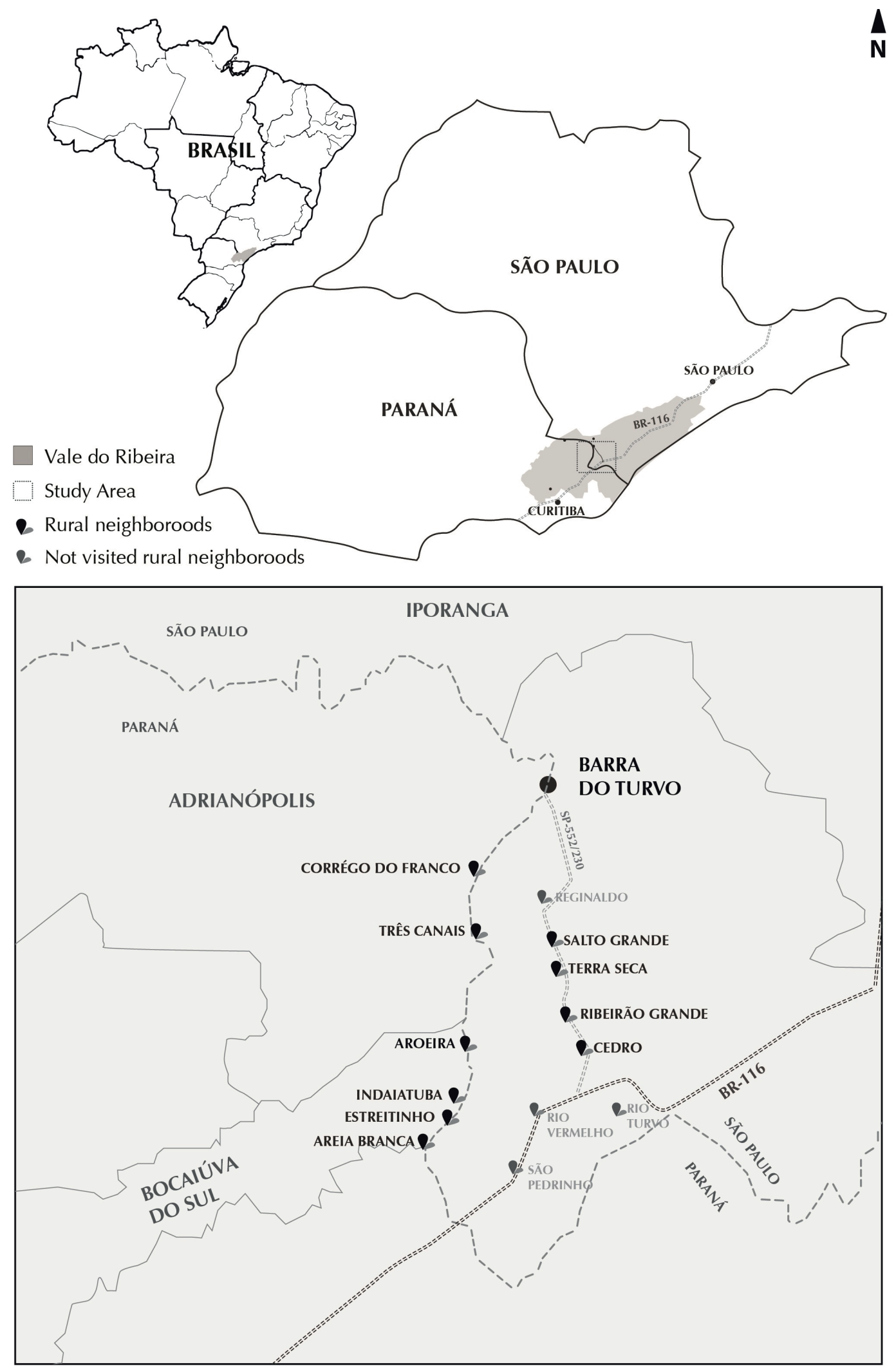

ALTO VALE DO RIBEIRA

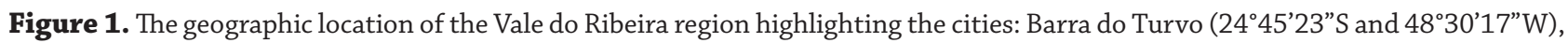
Adrianópolis ( $24^{\circ} 41^{\prime} 3^{\prime \prime} \mathrm{S}$ and $\left.48^{\circ} 58^{\prime} 12^{\prime \prime} \mathrm{W}\right)$ and Bocaiuva do Sul $\left(25^{\circ} 12^{\prime} 22^{\prime \prime} \mathrm{S}\right.$ and $\left.49^{\circ} 06^{\prime} 54^{\prime \prime} \mathrm{W}\right)$ as well as the respective rural districts where the member families of Cooperafloresta live (Image: Zé Diniz, 2014). 
and provided social and economic advantages to the association's members (Steenbock et al. 2013).

The Cooperafloresta is headquartered in Barra do Turvo, a small town $360 \mathrm{~km}$ from the city of São Paulo whose total population is 7,729 inhabitants who predominantly live in the rural areas (59 \%) (IBGE 2010). Approximately $22.62 \%$ of the residents are illiterate, and the average monthly rural income is $\mathrm{R} \$ 1.068,51$ (IBGE Cidades 2016). The main access to the area is a highway that is approximately $30 \mathrm{~km}$ from the town centre. Public transportation serves this stretch, whereas the rural districts are served by school buses that provide transportation to the city centre. The first public school was established in 1972 and offered only a basic education, whereas, today, the city has eight public schools that offer preschool, primary and secondary school including youth and adult education (unpubl. res.), a health centre and health professionals that serve people in rural districts.

Cooperafloresta consists of family farmers and, especially, quilombolas". Quilombolas are descendant populations of black slaves who currently perform activities related to agriculture, handicrafts, collecting and fishing (Diegues \& Arruda 2001). Barra do Turvo contains a nucleus of remaining quilombolas whose land is measured in Conservation Units (UCs). These are the Sustainable Development Reserves (RDS) of BarreiroAnhemas, Quilombos of Barra do Turvo and Pinheirinhos: places where traditional people can maintain their way of life as long as they use natural resources sustainably (Brasil 2000). Other quilombola nuclei in the region were granted legal land rights recognition by INCRA (the National Institute of Colonization and Agrarian Reform). These form communities in the neighbourhoods of Pedra Preta, Cedro, Ribeirão Grande/Terra Seca and Reginaldo (Andrade \& Tatto 2013).

The members are divided into groups of at least five families in accordance with the rural neighbourhoods where they live. Each group is responsible for choosing a board representative and holding monthly meetings and weekly collective work. Elected representatives represent the group in discussions and decisions at board meetings and are also responsible for disseminating information resulting from the meetings. The collective work can involve either physical labour or training. Collective work is a joint effort that promotes mutual aid among the farmers in the management of their land (Devide et al. 2013) and is conducted in different places. The training efforts are facilitated by the technical body that supports the organization and targeted to develop improved production strategies, commercialization and product certification.

Generally, members are spouses who are jointly involved in managing the agroforestry activities, but other family members may also be involved. In some cases, members' children are also members of the association and have their own management areas, but they also help their parents. The membership requirements involve practicing agroforestry, avoiding the burning of vegetation or the use of pesticides and chemical fertilizers, and participating in training and management activities, collective work and management of the Cooperafloresta. However, new members are permitted only upon the joint approval of the working group and the Council. Member families are also responsible for preparing agroforest management plans; they can count on technical support to achieve this outcome. Among the administrative procedures, self-management of resources is prioritized at both association scope association and individually (agroforestry). Farmers can hold positions at all levels except the technical positions.

\section{Informant profiles}

The 40 interviewed farmers, 17 women and 23 men, ranged from 18 to 78 years of age and belong to 23 family units with a mean age of 50 years (Fig. 2). Approximately $78 \%$ of the participants have lived in the area since they were born; another $18 \%$ come from nearby rural areas and $4 \%$ from northeastern Brazil. Regarding education, $42 \%$ attended up to the fourth grade of basic school, $25 \%$ had no schooling, $20 \%$ completed the eighth grade of basic school and $12 \%$ completed high school. Agroforestry production is the exclusive source of income for $20 \%$ of the sampled households. The other families, in addition to agricultural production, may have incomes supplemented by the government $(65 \%)$ including retirement, family allowance and pension, hold paid jobs such as cattle farming, beekeeping, public services, tourism and housecleaning or earn income from self-employment (35\%).

\section{Ethnobotanical data collection}

Meetings were held to present the study proposal and answer questions to obtain prior consent of the association and meet legal procedures involving authorization to access local knowledge (IPHAN authorization No. 01450.004903 /2013-5). Members of the Cooperafloresta who were over 18 years of age and agreed to sign the Free and Informed Consent Term were permitted to participate in the survey. The participants were selected through non-probabilistic sampling using the "snowball" technique (Tongco 2007). The first participants $(n=13)$ were suggested by the technical staff of the association because they were able to answer the survey questions. In turn, these first participants were asked to refer other farmers. The spouses and children of the first participants were contacted as well because information regarding the use of plants may not be shared among the people in the same household (Araújo et al. 2012).

The sampling effort was assessed by a rarefaction curve associated with the Jacknife 1 index (Williams et al. 2007) and compiled from data generated by EstimateS 9.0 software (Collwell 2013). Rarefaction curves in ethnobotany 


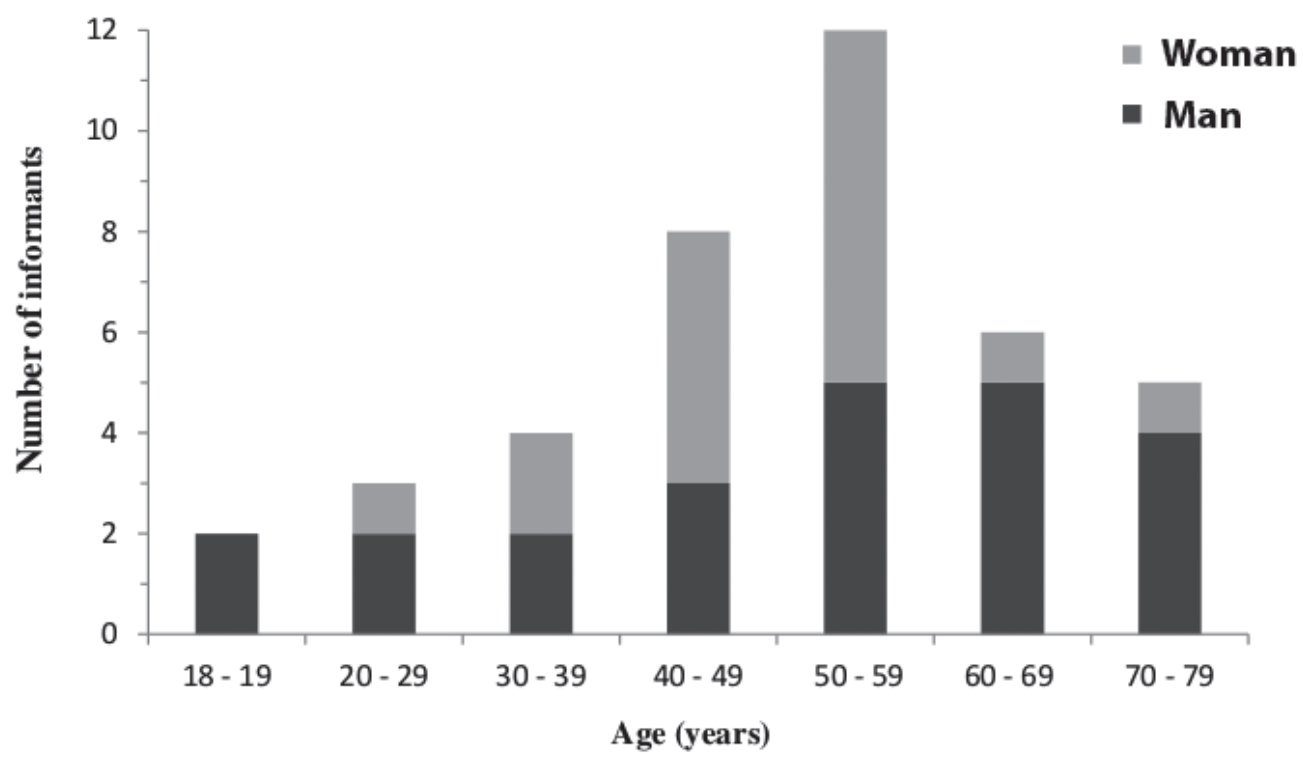

Figure 2. Gender distribution in relation to the age groups of the interviewed farmers, members of Cooperafloresta, and the Vale do Ribeira, Brazil.

studies show that the sampling effort is sufficient when few new species are added by completing new interviews (Begossi 1996). Thus, this procedure was conducted for the population in general; a total of 40 participants ( 23 men and 17 women) were required for the effort to be sufficient. The Jacknife 1 index is used to reduce the underestimation of the true number of species in a single sample and is based on the presence of a species in a single sample unit (Collwell \& Coddington 1994). In this case, it was possible to calculate that approximately $80 \%$ of the expected richness (Sobs = 218 and Sest $=272.6$ ) was sampled after the 40 interviews were conducted (Fig. 3).

Data collection occurred between July 2012 and December 2013 and involved semi-structured interviews (a socioeconomic questionnaire and a free list), guided tours and direct observations (Albuquerque et al. 2014). The socioeconomic questionnaire aimed to define participant profiles based on provided data such as age, gender, occupation, source of income and period of residence. On the free list, the participants were asked to indicate all useful and locally known trees. Based on this list, in a second meeting with the participants, specific questions were asked regarding each of the mentioned plants and concerning their uses. The guided tour consisted of a walk with all participants through yards, gardens, trails and woods where they could encounter the largest number of plants mentioned in the free list. The plant specimens were photographed and, whenever possible, were collected for taxonomic identification and herborization. Additionally, the direct observations were combined with records of the phenomena observed in the field to support the qualitative analyses.

\section{Data analysis}

The plants included in the free list were analysed according to their popular names and considering ethnospecies (Hanazaki et al. 2000). When one or more ethnospecies referred to a single botanical species, only one popular name was recorded. The identification of the collected plant material was performed by comparison with herbarium material and by consulting with specialists and through support from the literature. The reference material in the Herbarium Maria Fidalgo Eneyda P. K.-Instituto de Botânica, São Paulo, SP, Brazil, was indicated for each identified species. This procedure was adopted because most specimens are sterile or belong to common species already deposited in the herbarium.

The species on the list were grouped into nine use categories: food, animal feed, construction, fuel, organic matter, medicines, beekeeping, technology and others according to Ramos et al. (2014) and Cunha \& Albuquerque (2006) and analysed based on the frequency with which they were mentioned. The participants were classified according to gender (men and women) and age group to identify differences in knowledge between groups. For the age-based categorization, the classification used by the Brazilian institute of geography and statistics-IBGE (2010) — was adopted: $15-24$ years (young person, $n=4$ ), 25-59 years (adult, $\mathrm{n}=25$ ) and above 60 years (elderly, $\mathrm{n}=$ 


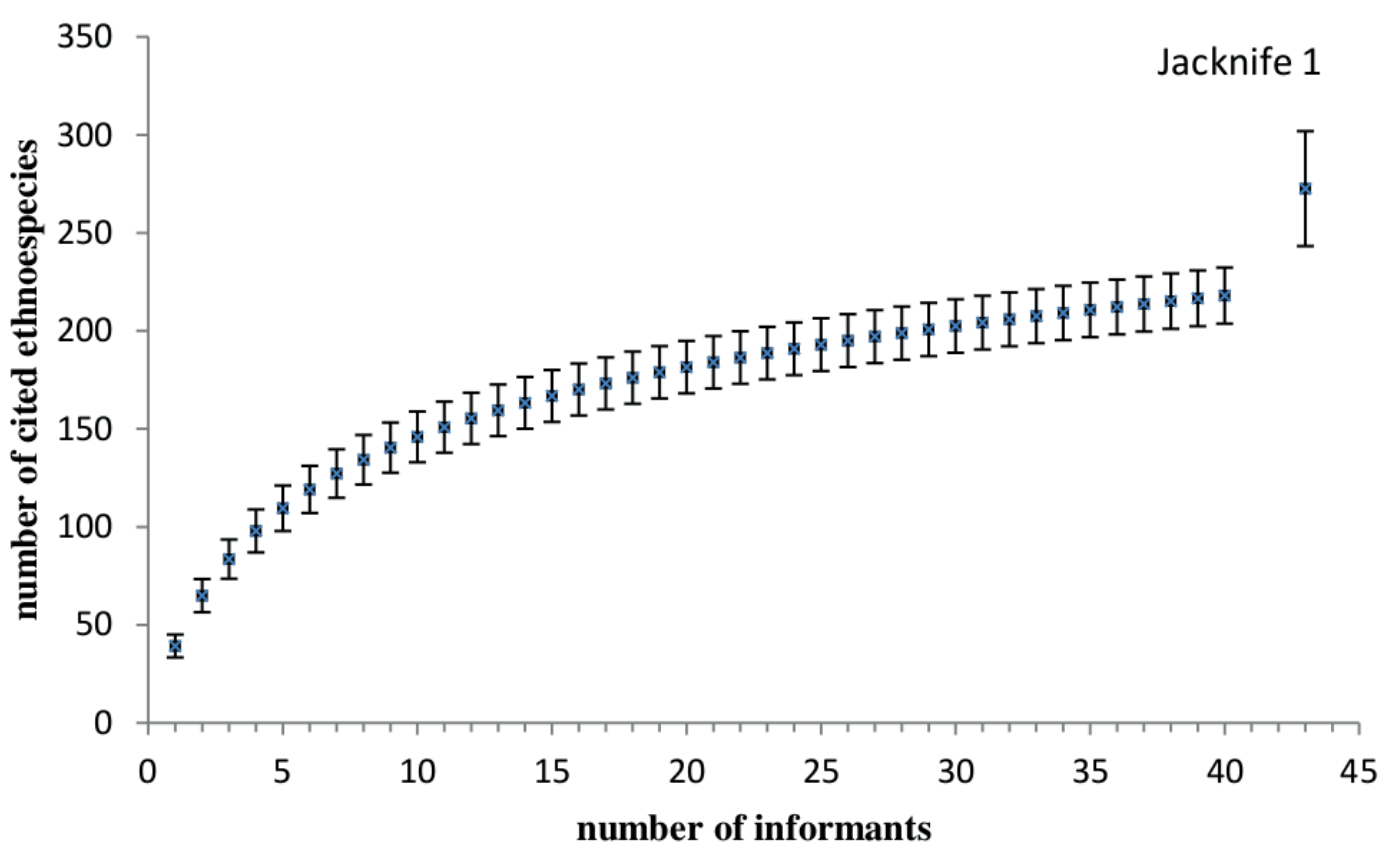

Figure 3. A rarefaction curve containing the number of cited ethnospecies and the number of interviews conducted with member farmers of Cooperafloresta, Ribeira Valley, Brazil. Confidence intervals are at the $95 \%$ level, and associations with the wealth estimator used Jacknife 1.

11). The participants' knowledge was analysed based on the total number and average of the mentioned species and the number of native, exotic and species reported exclusively by each group. To determine whether the variables were significantly different, Kruskal-Wallis tests were applied to the average number of species mentioned by men and women and between young people, adults and the elderly, while chi-square tests were used for the total number of listed species. Both statistical procedures were performed using BioEstat 5.0 software (Ayres et al. 2007).

\section{Results}

\section{Knowledge in relation to gender}

Knowledge did not differ between the genders with regard to the total richness of known species $\left(X^{2}=1.94, \mathrm{p}\right.$ $=0.18)$-men mentioned $173(44.13 \pm 17.33)$ and women mentioned $148(34.76 \pm 09.10)$ species (Tab. 1). When analysing the differences between their knowledge in the respective use categories, as shown in Tab. 2, men mentioned more species in the beekeeping category $\left(X^{2}=5.78, p=\right.$ 0.02 ) and the mean values of species used in construction mentioned by men were higher $(H=6.64, p=0.09)$. However, the number of species mentioned exclusively by men (36 sp.) was higher than that mentioned by women (11 sp.) $\left(X^{2}=13.29, \mathrm{p}=0.0005\right)$. Considering the plants exclusively reported by each gender, men mentioned significantly higher species richness in the construction $\left(X^{2}=13.44, \mathrm{p}=0.0005\right)$, organic matter $\left(X^{2}=4.78, \mathrm{p}=0.04\right)$, fuel $\left(X^{2}=7.75, \mathrm{p}=\right.$ $0.009)$, animal feed $\left(X^{2}=5.56, p=0.02\right)$, technology $\left(X^{2}=\right.$ $8.25, \mathrm{p}=0.006)$ and beekeeping $\left(X^{2}=10.80, \mathrm{p}=0.0019\right)$ categories, whereas in the food $\left(X^{2}=2.33, p=0.19\right)$, medical $\left(X^{2}=1.38, \mathrm{p}=0.326\right)$ and other categories $\left(X^{2}=2.61, \mathrm{p}=\right.$ $0.15)$, there were no significant differences between genders. The 11 species listed solely by women were related to their uses as food ( $6 \mathrm{sp}$.), feed (3 sp.), construction (1 sp.), organic matter ( $1 \mathrm{sp}$.$) , medicine (1 sp.) and others ( 1 \mathrm{sp}$.$) .$

\section{Knowledge in relation to age}

The total species richness was $74(33.25 \pm 20.55)$ by young people, $163(39.08 \pm 12.96)$ by adults and $150(45.09 \pm 18.19)$ by the elderly. Statistically, significant differences were found when young people were compared with adults $\left(X^{2}=33.42\right.$, $\mathrm{p}<0.0001)$ and with the elderly $\left(X^{2}=30.12, \mathrm{p}<0.0001\right)$, indicating that the species richness familiar to participants aged 18-24 is below that of adults and the elderly. However, adults and the elderly $\left(X^{2}=0.54, \mathrm{p}=0.49\right)$ mentioned a similar number of useful trees. The analysis of the influence of age in terms of knowledge of use categories (Tab. 3) revealed that adults mentioned more species employed as organic matter, fuel and medicine than did young people $\left(X^{2}\right.$ $=33.13, \mathrm{p}<0.0001 ; X^{2}=35.83, \mathrm{p}<0.0001 ; X^{2}=45.06, \mathrm{p}<$ 0.0001 , respectively) and the elderly $\left(X^{2}=13.88, \mathrm{p}=0.0003\right.$; $X^{2}=7.51, \mathrm{p}=0.0084 ; X^{2}=6.54, \mathrm{p}=0.01$, respectively). The elderly mentioned higher average values of species used for technology than did young people $(\mathrm{H}=7.58, \mathrm{p}=0.005)$ or adults $(\mathrm{H}=4.46, \mathrm{p}=0.03)$. 


\section{Do gender and age influence agroforestry farmers' knowledge of tree species uses in an area of the Atlantic Forest, Brazil?}

Table 1. A list of tree species cited as useful by the agroforestry farmers of Barra do Turvo and Adrianópolis in the Vale do Ribeira, Brazil, showing the botanical families, scientific and local names in alphabetical order and their use categories: food (Fd), animal feed (Af), construction (C), fuel ( $\mathrm{Fl}$ ), organic matter (Or), medicines (M), beekeeping (B), technology (T) and others (O). Legend: * Synonyms - when one or more ethnospecies refers to a single botanical species. ${ }^{* *}$ Homonyms (type 1 ) - when an ethnospecies refers to two or more plant species of the same genus. ${ }^{* * *}$ Homonyms (type 2 )-when an ethnospecies refers to two or more plant species of different types.

\begin{tabular}{|c|c|c|c|}
\hline Botanical families/ Scientific name & Local name & Origin & Use categories \\
\hline \multicolumn{4}{|l|}{ ACANTHACEAE } \\
\hline Aphelandra liboniana Linden ex Hook. & Balsamo de bugre & $\mathrm{N}$ & M \\
\hline \multicolumn{4}{|l|}{ ADOXACEAE } \\
\hline Sambucus australis Cham. \& Schltdl. & Sabugueiro & $\mathrm{N}$ & Or, M \\
\hline \multicolumn{4}{|l|}{ ANCARDIACEAE } \\
\hline Astronium graveolens Jacq. & Pau amargo, pau tenente & $\mathrm{N}$ & C, Or, M \\
\hline Anacardium occidentale L. & Caju & $\mathrm{N}$ & Fd, M \\
\hline Lithrea molleoides (Vell.) Engl. & Aroeira** $^{* *}$ & $\mathrm{~N}$ & Af, C, Fl, Or, M, O \\
\hline Schinus terebinthifolia Raddi & & $\mathrm{N}$ & \\
\hline Mangifera indica L. & Manga & E & Fd, Or, M, O \\
\hline Myracrodruon urundeuva Allemão & Méssica & $\mathrm{N}$ & $\mathrm{M}, \mathrm{O}$ \\
\hline Spondias dulcis Parkinson & Cajá manga & E & Fd, C, Or \\
\hline Spondias mombin L. & Cajá mirim & $\mathrm{N}$ & Fd, Or, B \\
\hline Spondias purpurea L. & Ciriguela & $\mathrm{E}$ & Fd, B \\
\hline \multicolumn{4}{|l|}{ ANNONACEAE } \\
\hline Annona emarginata (Schltdl.) H. Rainer & Ariticum, araticum* & $\mathrm{N}$ & Fd, Fl, C, Or, T \\
\hline Annona muricata L. & Graviola & $\mathrm{E}$ & Fd, Or \\
\hline Annona squamosa L. & Fruta do conde, ata* & E & $\mathrm{Fd}$ \\
\hline Annona sylvatica A.St.-Hil. & Ariticum duro & $\mathrm{N}$ & Fd \\
\hline Duguetia lanceolata A.St.-Hil. & Graviola azeda, graviola doce* & $\mathrm{N}$ & $\mathrm{Fd}, \mathrm{Or}$ \\
\hline \multicolumn{4}{|l|}{ APOCYNACEAE } \\
\hline Aspidosperma polyneurum Müll. Arg. & Guatambu perova, perovinha* & $\mathrm{N}$ & $C, \mathrm{~T}$ \\
\hline Aspidosperma subincanum Mart. & Guatambu & $\mathrm{N}$ & $\mathrm{Fl}, \mathrm{C}, \mathrm{T}$ \\
\hline Tabernaemontana catharinensis A. DC. & Goriana, gueriana* & $\mathrm{N}$ & C \\
\hline \multicolumn{4}{|l|}{ AQUIFOLIACEAE } \\
\hline Ilex paraguariensis A. St.-Hil. & Erva mate & $\mathrm{N}$ & $\mathrm{Fd}$ \\
\hline \multicolumn{4}{|l|}{ ARALIACEAE } \\
\hline Dendropanax cf. cuneatus (DC.) Decne. \& Planch. & Caruru, embu** & $\mathrm{N}$ & Af, Fd, Or \\
\hline \multicolumn{4}{|l|}{ ARAUCARIACEAE } \\
\hline Araucaria angustifolia (Bertol.) Kuntze & Pinheiro, araucária* & $\mathrm{N}$ & $\mathrm{Fd}, \mathrm{C}, \mathrm{M}, \mathrm{T}$ \\
\hline \multicolumn{4}{|l|}{ ARECACEAE } \\
\hline Archontophoenix alexandrae (F. Muell.) H. Wendl. \& Drude & Palmeira real & $\mathrm{E}$ & Fd \\
\hline Astrocaryum aculeatissimum (Schott) Burret & Brajaúva, bajarova* & $\mathrm{N}$ & Af, Fd, C, T \\
\hline Bactris gasipaes Kunth & Pupunha & $\mathrm{N}$ & Af, Fd, C, Or, B \\
\hline Bactris setosa Mart. & Tucum & $\mathrm{N}$ & Fd, Fl, T \\
\hline Cocos nucifera L. & Coco & $\mathrm{E}$ & $\mathrm{Fd}$ \\
\hline Euterpe edulis Mart. & Juçara & $\mathrm{N}$ & Af, Fd, Or \\
\hline Geonoma elegans Mart. & Guaricana & $\mathrm{N}$ & Or \\
\hline Syagrus romanzoffiana (Cham.) Glassman & Jerivá, jarová* & $\mathrm{N}$ & Af, Fd, B \\
\hline \multicolumn{4}{|l|}{ ASTERACEAE } \\
\hline Baccharis cf. semiserrata DC. & Tapixaba, alecrim, vassoreira* & $\mathrm{N}$ & Or, Fl, M, B \\
\hline Vernonia densiflora Gardner & Assa peixe & $\mathrm{N}$ & $\mathrm{Fl}, \mathrm{Or}, \mathrm{M}, \mathrm{B}$ \\
\hline \multicolumn{4}{|l|}{ BIGNONIACEAE } \\
\hline Handroanthus chrysotrichus (Mart. Ex A. DC.) Mattos & Ipê amarelo** & $\mathrm{N}$ & C, Or, M, Or, T \\
\hline Handroanthus serratifolius (A.H.Gentry) S.Grose & & $\mathrm{N}$ & \\
\hline Handroanthus impetiginosus (Mart. ex DC.) Mattos & Ipê roxo** & $\mathrm{N}$ & C, M, Or, T \\
\hline Handroanthus cf. ochraceus (Cham.) Mattos & & $\mathrm{N}$ & \\
\hline Jacaranda micrantha Cham. & Carova, varova* & $\mathrm{N}$ & Af, Fl, C, M, B. Or, T \\
\hline \multicolumn{4}{|l|}{ BIXACEAE } \\
\hline Bixa orellana L. & Coloral, urucum* & $\mathrm{N}$ & Fd, Or, M, O \\
\hline \multicolumn{4}{|l|}{ BORAGINACEAE } \\
\hline Cordia magnoliifolia Cham. & Erva drinda & $\mathrm{N}$ & Af \\
\hline
\end{tabular}


Table 1. Cont.

Botanical families/ Scientific name

CANNABACEAE

Trema micrantha (L.) Blume

CELASTRACEAE

Maytenus ilicifolia Mart. ex Reissek

CLUSIACEAE

Garcinia cochinchinensis Choisy

Garcinia gardneriana (Planch. \& Triana) Zappi

CONNARACEAE

Connarus $s p$.

EBENACEAE

Diospyros kaki Thunb.

ELAEOCARPACEAE

Sloanea guianensis (Aubl.) Benth.

EUPHORBIACEAE

Alchornea glandulosa Poepp. \& Endl.

Croton floribundus Spreng.

Croton urucurana Baill

Maprounea brasiliensis A.St.-Hil.

Maprounea guianensis Aubl.

Sapium glandulosum (L.) Morong

FABACEAE

Anadenanthera colubrina (Vell.) Brenan

Arachis hypogaea L.

Bauhinia blakeana Dunn.

Caesalpinia echinata Lam.

Carya illinoinensis (Wangenh.) K. Koch

Cassia ferruginea (Schrad.) Schrad. ex DC.

Centrolobium tomentosum Guillemin ex Benth.

Tachigali cf. multijuga Benth.

Copaifera langsdorffii Desf.

Dahlstedtia pinnata (Benth.) Malme

Dinizia excelsa Ducke

Enterolobium contortisiliquum (Vell.) Morong

Senna multijuga (Rich.) H.S.Irwin \& Barneby

Erythrina crista-galli L.

Erythrina sp.

Erythrina speciosa Andrews

Hymenaea courbaril L.

Inga spp.

Inga edulis Mart.

Inga sessilis (Vell.) Mart.

Inga marginata Willd.

Leucaena leucocephala (Lam.) R. de Wit.

Lonchocarpus muehlbergianus Hassl.

Machaerium nyctitans (Vell.) Benth.

Machaerium stipitatum Vogel

Mimosa scabrella Benth.

Myrocarpus frondosus Allemão

Myroxylon peruiferum L.f.

Ormosia arborea (Vell.) Harms

Piptadenia gonoacantha (Mart.) J.F. Macbr.

Piptadenia paniculata Benth.

Schizolobium parahyba (Vell.) Blake

Swartzia submarginata (Benth.) Mansano

Vitex polygama Cham.
Local name

Origin

Use categories

Crandiuva, carandiuva, canjuva*

Espinheira santa

$\mathrm{N}$

$\mathrm{N}$

Mangustão

E

Vacupari

$\mathrm{N}$

Amarelinho**

Caqui

E

Cabriteira

$\mathrm{N}$

Tapiá, tapiaeiro*

Tapixingui, capixingui*

Sambueiro

Sucareiro

Amarelino**

Pica cu, leiteiro*

Angico, gorocaia*

Amendoim

Pata de vaca

Pau Brasil

Noz Pecã

Canafrista, quaresmeira*

Aririvá, ararivá, araribá, arivá**

Copaíba

Cabreuva

Angelim

Caquera**

Corticeira brejo

Corticeira, cortumeiro, mulungu*

Eritrina

Jatobá, Jataí*

Ingá

Ingá cipó, ingá de metro*

Ingá macaco, ingá branco*

Ingá mirim, ingá feijão*

Leucena

Imbira caboclo, timboeiro, timbó*

Bico de pato

Carandá, sapuva, asa de grilo*

Bracatinga

Cabureira, caburê, cabuí*

Jacarandá

Olho de cabra

Jacaré

Mamica de cabela

Guapiruvu

Guê

Tarumã
Af, Fl, Or, O

M

$\mathrm{Fd}$

Fd, C, Or

Af, Fl, C, Or, T

Fd, Or

Fl, C

Fl, C, Or, B, T

Af, Fl, C, Or, B

$\mathrm{Or}, \mathrm{Fl}$

Or

Af, Fl, C, Or

Or, B, O

Fl, C, Or, M, B, O

Fd

$\mathrm{M}, \mathrm{Fl}$

C

$\mathrm{Fd}$

Fl, C, B, Or, T

Fl, C, Or, T

Af, C, M, T

C

C, B, T

C, $\mathrm{M}, \mathrm{B}$

Or

Or, M, B, O

Or, O

Af, Fd, Fl, C, M, T

$\mathrm{Fd}, \mathrm{Fl}, \mathrm{Or}, \mathrm{B}$

Af, Fd, Fl, Or, B

Af, Fd, C, Or, B, O

Af, Fd, C, Or, B, O

Fl, C, Or

$\mathrm{Fl}, \mathrm{C}, \mathrm{Or}, \mathrm{T}, \mathrm{O}$

Af, Fl, C, Or, B, T

$\mathrm{Fl}, \mathrm{C}, \mathrm{T}$

Af, Fl, C, Or, B, T

C

Fd, Fl, C, Or, B, T

Or

$\mathrm{Fl}, \mathrm{C}, \mathrm{Or}, \mathrm{M}$

Fl, Or, M

Fl, C, Or, T, O

Fl, C

Af, Fd, Fl, C, M 
Table 1. Cont.

\begin{tabular}{|c|c|c|c|}
\hline Botanical families/Scientific name & Local name & Origin & Use categories \\
\hline \multicolumn{4}{|l|}{ LAURACEAE } \\
\hline cf. Cinnamomum sp. & Canela** & & $\mathrm{Fl}, \mathrm{C}, \mathrm{T}$ \\
\hline Nectandra megapotamica (Spreng.) Mez & & $\mathrm{N}$ & \\
\hline Cinnamomum triplinerve (Ruiz \& Pav.) Kosterm. & Canela amarela & $\mathrm{N}$ & Af, C, Or, B, T \\
\hline Cryptocarya sp. & Canela iutinga, canela niutinga* & & Af, Fl, C, M \\
\hline Nectandra membranacea (Sw.) Griseb. & Canela preta & $\mathrm{N}$ & C, $\mathrm{T}$ \\
\hline Ocotea brachybotrya (Meisn.) Mez & Canela feijão & $\mathrm{N}$ & $C$ \\
\hline Ocotea prolifera (Nees \& Mart.) Mez & $\begin{array}{l}\text { Canela sassafrás, canela cravo, canela } \\
\text { de cheiro** }\end{array}$ & $\mathrm{N}$ & $\mathrm{Fd}, \mathrm{C}, \mathrm{M}, \mathrm{T}$ \\
\hline Ocotea puberula (Rich.) Nees & $\begin{array}{l}\text { Canela niuva, canela sebo, canela } \\
\text { branca* }\end{array}$ & $\mathrm{N}$ & Af, Fl, C, Or, M, T \\
\hline Persea americana Mill. & Abacate & $\mathrm{E}$ & Fd, Fl, Or, M \\
\hline Persea willdenovii Kosterm. & Canela andrade & $\mathrm{N}$ & C, $\mathrm{M}$ \\
\hline \multicolumn{4}{|l|}{ LECYTHIDACEAE } \\
\hline Cariniana estrellensis (Raddi) Kuntze & Imbira caboclo branca & $\mathrm{N}$ & $\mathrm{O}$ \\
\hline \multicolumn{4}{|l|}{ LYTHRACEAE } \\
\hline Punica granatum $\mathrm{L}$. & Romã & E & $\mathrm{Fd}, \mathrm{M}$ \\
\hline \multicolumn{4}{|l|}{ MAGNOLIACEAE } \\
\hline Magnolia ovata (A. St.-Hil.) Spreng. & Maria mole, vaguaçu* & $\mathrm{N}$ & C \\
\hline \multicolumn{4}{|l|}{ MALPIGHIACEAE } \\
\hline Malpighia emarginata DC. & Acerola & E & Fd, Or \\
\hline \multicolumn{4}{|l|}{ MALVACEAE } \\
\hline Ceiba speciosa (A. St.-Hil.) Ravenna & Paineira & $\mathrm{N}$ & Af, Or \\
\hline Dombeya wallichii(Lindl.) Baill. & Atrapéia, estrapéia* & $\mathrm{E}$ & Or, B \\
\hline Luehea divaricata Mart. \& Zucc. & $\begin{array}{l}\text { Açoita cavalo, tamanqueira, } \\
\text { tucaneira* }\end{array}$ & $\mathrm{N}$ & Af, Fl, C, Or, M, B, T \\
\hline Malvaviscus arboreus Cav. & Sapateira & E & C \\
\hline Pachira glabra Pasq & Castanheira & E & Fd \\
\hline Pseudobombax grandiflorum (Cav.) A.Robyns & Embiruçu, imbiruçu* & $\mathrm{N}$ & Af, Or, O \\
\hline Theobroma cacao L. & Cacau, cacau amarelo* & E & Fd \\
\hline \multicolumn{4}{|l|}{ MELASTOMATACEAE } \\
\hline Tibouchina fothergillae (Schrank \& Mart. ex DC.) Cogn. & Jacatirão, flor de maio, natal ${ }^{* *}$ & $\mathrm{~N}$ & $\mathrm{Fl}, \mathrm{C}, \mathrm{Or}, \mathrm{B}, \mathrm{O}$ \\
\hline Tibouchina mutabilis (Vell.) Cogn. & & $\mathrm{N}$ & \\
\hline \multicolumn{4}{|l|}{ MELIACEAE } \\
\hline Cabralea canjerana (Vell.) Mart. & Cajarana, canharanda* & $\mathrm{N}$ & Af, $C$ \\
\hline Cedrela fissilis Vell. & Cedro, cedro branco* & $\mathrm{N}$ & C, Or, M, T, O \\
\hline Khaya senegalensis (Desr.) A. Juss. & Mogno africano & $\mathrm{E}$ & C \\
\hline Melia azedarach L. & Espinheira santa, santa barbara** & E & Af, Fl, C, T \\
\hline Swietenia macrophylla King & Mogno & $\mathrm{N}$ & C \\
\hline Toona ciliata M.Roem. & Cedro australiano & $\mathrm{E}$ & C \\
\hline \multicolumn{4}{|l|}{ MORACEAE } \\
\hline Artocarpus heterophyllus Lam. & Jaca & E & Af, Fd, Fl, C, Or, M, O, T \\
\hline Ficus luschnathiana (Miq.) Miq. & Figueira branca & $\mathrm{N}$ & Af, C, Or \\
\hline Maclura tinctoria (L.) D. Don ex Steud. & Taiuva, amoreira branca* & $\mathrm{N}$ & Af, Fd, C, Or, M \\
\hline Morus nigra L. & Amora & $\mathrm{E}$ & Af, Fd, C, Or, M, O \\
\hline \multicolumn{4}{|l|}{ MUNTINGIACEAE } \\
\hline Muntingia calabura L. & Calabura & $\mathrm{N}$ & Af, Fd \\
\hline \multicolumn{4}{|l|}{ MYRISTICACEAE } \\
\hline Virola cf. bicuhyba (Schott ex Spreng.) Warb. & Bucuveira** $^{* *}$ & $\mathrm{~N}$ & Af, C, M, Or \\
\hline \multicolumn{4}{|l|}{ MYRTACEAE } \\
\hline Campomanesia neriiflora (O.Berg) Nied. & Gabiroba miúda & $\mathrm{N}$ & Fd, C, M, T \\
\hline Campomanesia phaea (O.Berg) Landrum & Cambuci & $\mathrm{N}$ & $\mathrm{Fd}$ \\
\hline Campomanesia xanthocarpa (Mart.) O.Berg & $\begin{array}{l}\text { Gabiroba, gabiroba amarela, gabiroba } \\
\text { de porco }\end{array}$ & $\mathrm{N}$ & Af, Fd, Fl, C, Or, M, B, T \\
\hline Eucalyptus sp. & Eucalipto & E & $\mathrm{Fl}, \mathrm{C}, \mathrm{Or}, \mathrm{M}$ \\
\hline Eugenia beaurepairiana (Kiaersk.) D.Legrand & Murta & $\mathrm{N}$ & Af, Fd \\
\hline
\end{tabular}


Table 1. Cont.

\begin{tabular}{|c|c|c|c|}
\hline Botanical families/Scientific name & Local name & Origin & Use categories \\
\hline Eugenia brasiliensis Lam. & Grumixama & $\mathrm{N}$ & Fd \\
\hline Eugenia florida DC. & Camarinho & $\mathrm{N}$ & Af, Fd, Fl, C, Or, B \\
\hline Eugenia involucrata DC. & Cereja & $\mathrm{N}$ & Af, Fd, C, B \\
\hline Eugenia pyriformis Cambess. & Uvaia, ovalha** & $\mathrm{N}$ & Fd \\
\hline Psidium myrtoides O.Berg & & $\mathrm{N}$ & \\
\hline Eugenia sp. & Vatinga & & C \\
\hline Eugenia uniflora L. & Pitanga & $\mathrm{N}$ & Fd, M, B, Or \\
\hline Myrcia splendens (Sw.) DC. & Guamirim & $\mathrm{N}$ & Fl, B \\
\hline Myrciaria glazioviana (Kiaersk.) G.M.Barroso ex Sobral & Cabeludinha & $\mathrm{N}$ & Fd, Af \\
\hline Pimenta pseudocaryophyllus (Gomes) Landrum & $\begin{array}{l}\text { Canela sassafrás, canela cravo, canela } \\
\text { de cheiro** }\end{array}$ & $\mathrm{N}$ & $\mathrm{Fd}, \mathrm{C}, \mathrm{M}, \mathrm{T}$ \\
\hline Plinia peruviana (Poir.) Govaerts & Jabuticaba & $\mathrm{N}$ & Fd, M \\
\hline Psidium cattleianum Sabine & Araçá** & $\mathrm{N}$ & Af, Fd, Fl, C, Or, M \\
\hline Psidium cf. rufum DC. & & $\mathrm{N}$ & \\
\hline Psidium guajava L. & Goiaba & $\mathrm{E}$ & Fd, Fl, C, Or, B \\
\hline Syzygium cumini (L.) Skeels & Jambolão & $\mathrm{E}$ & Af, Fd \\
\hline Syzygium jambos (L.) Alsto & Jambo, jambre* & $\mathrm{E}$ & Fd \\
\hline \multicolumn{4}{|l|}{ OLACACEAE } \\
\hline Tetrastylidium grandifolium (Baill.) Sleumer & Mandigaru & $\mathrm{N}$ & C \\
\hline \multicolumn{4}{|l|}{ OXALIDACEAE } \\
\hline Averrhoa carambola L. & Carambola & $\mathrm{E}$ & Af, Fd, B \\
\hline \multicolumn{4}{|l|}{ PERACEAE } \\
\hline Pera glabrata (Schott) Poepp. ex Baill. & Bucuveira** & $\mathrm{N}$ & Af, C, M, Or \\
\hline \multicolumn{4}{|l|}{ PHYLLANTHACEAE } \\
\hline Hieronyma alchorneoides Allemão & Urucurana, urucuveira, nicurana* & $\mathrm{N}$ & Af, Fd, Fl, C, Or, O, T \\
\hline \multicolumn{4}{|l|}{ PHYTOLACCACEAE } \\
\hline Gallesia integrifolia (Spreng.) Harms & Guararema, pau d'alho* & $\mathrm{N}$ & $\mathrm{Fd}, \mathrm{C}, \mathrm{Or}, \mathrm{O}, \mathrm{T}$ \\
\hline Phytolacca dioica $\mathrm{L}$. & Caruru, embu** & $\mathrm{N}$ & Af, Fd, Or \\
\hline Seguieria langsdorffii Moq. & Limoeiro bravo & $\mathrm{N}$ & Or, B \\
\hline \multicolumn{4}{|l|}{ PIPERACEAE } \\
\hline Piper aduncum L. & Jaborandi** & $\mathrm{N}$ & Af, Fd, Fl, Or, M \\
\hline Piper arboreum Aubl. & & $\mathrm{N}$ & \\
\hline \multicolumn{4}{|l|}{ PRIMULACEAE } \\
\hline Myrsine cf. lancifolia Mart. & Pororoca, capororoca* & $\mathrm{N}$ & Af, $\mathrm{Fl}, \mathrm{C}, \mathrm{Or}$ \\
\hline \multicolumn{4}{|l|}{ PROTEACEAE } \\
\hline Roupala montana cf. var. brasiliensis (Klotzsch) K.S.Edwards & Carvalho & $\mathrm{N}$ & C, M, T \\
\hline \multicolumn{4}{|l|}{ RHAMNACEAE } \\
\hline Hovenia dulcis Thunb. & Uva japão & $\mathrm{E}$ & Af, Fd, Fl, C, Or, B \\
\hline \multicolumn{4}{|l|}{ ROSACEAE } \\
\hline Eriobotrya japonica (Thunb.) Lindl. & Ameixa & $\mathrm{E}$ & Af, Fd, Fl, M, B \\
\hline Malus x domestica Borkh. & Maçã & E & $\mathrm{Fd}$ \\
\hline Prunus myrtifolia (L.) Urb. & Pêssegueiro bravo & $\mathrm{N}$ & $\mathrm{C}, \mathrm{O}$ \\
\hline Prunus persica (L.) Batsch & Pêssego & E & Fd, Or, M, B \\
\hline Pyrus communis $\mathrm{L}$. & Pêra & $\mathrm{E}$ & Fd \\
\hline \multicolumn{4}{|l|}{ RUBIACEAE } \\
\hline Bathysa australis (A. St.-Hil.) Hook. f. ex K. Schum. & João henrique & $\mathrm{N}$ & Or \\
\hline Coffea arabica L. & Café & $\mathrm{E}$ & $\mathrm{Fd}, \mathrm{M}$ \\
\hline Coutarea hexandra (Jacq.) K.Schum & Quina amarela & $\mathrm{N}$ & Af, C, M, T \\
\hline Genipa americana L. & Jenipapo & $\mathrm{N}$ & $\mathrm{Fd}$ \\
\hline Posoqueria latifolia (Rudge) Schult. & Fruto de macaco, limão macaco* & $\mathrm{N}$ & $\mathrm{Fd}$ \\
\hline Psychotria suterella Müll. Arg. & Alface de anta & $\mathrm{N}$ & Af \\
\hline \multicolumn{4}{|l|}{ RUTACEAE } \\
\hline Citrus $x$ aurantiifolia (Christm.) Swingle & Lima da pérsia, limão galego* & E & $\mathrm{Fd}$ \\
\hline Citrus $\times$ latifolia Tanaka ex Q. Jiménez & Limão taiti & E & Fd \\
\hline Citrus limon (L.) Osbeck & Limão, limoeiro & E & Fd, M, B \\
\hline Citrus $x$ limonia Osbeck & Limão rosa & $\mathrm{E}$ & Fd \\
\hline
\end{tabular}


Table 1. Cont.

\begin{tabular}{|c|c|c|c|}
\hline Botanical families/ Scientific name & Local name & Origin & Use categories \\
\hline Citrus reticulata Blanco & Mexirica & $\mathrm{E}$ & $\mathrm{Fd}, \mathrm{M}$ \\
\hline Citrus X sinensis (L.) Osbeck & Laranja, laranja bahia, laranja pêra* & $\mathrm{E}$ & $\mathrm{Fd}, \mathrm{Fl}, \mathrm{M}, \mathrm{B}$ \\
\hline \multicolumn{4}{|l|}{ SABIACEAE } \\
\hline Meliosma itatiaiae Urb. & Orelha de mula & $\mathrm{N}$ & C, Or, T \\
\hline \multicolumn{4}{|l|}{ SALICACEAE } \\
\hline Casearia cf. obliqua Spreng. & Erva de macuco, guaçatunga** & $\mathrm{N}$ & Fl, C, Or, M, O \\
\hline Casearia sylvestris Sw. & & $\mathrm{N}$ & \\
\hline \multicolumn{4}{|l|}{ SAPINDACEAE } \\
\hline Cupania oblongifolia Mart. & Migué pintado & $\mathrm{N}$ & $\mathrm{Fl}, \mathrm{C}, \mathrm{Or}, \mathrm{T}$ \\
\hline Litchi chinensis Sonn. & Lichia & $\mathrm{E}$ & $\mathrm{Fd}$ \\
\hline Matayba intermedia Radlk. & Caviuna & $\mathrm{N}$ & $\mathrm{C}, \mathrm{T}$ \\
\hline \multicolumn{4}{|l|}{ SAPOTACEAE } \\
\hline Chrysophyllum inornatum Mart. & Aleixo & $\mathrm{N}$ & $\mathrm{T}$ \\
\hline Pouteria caimito (Ruiz \& Pav.) Radlk. & Abiu & $\mathrm{N}$ & $\mathrm{Fd}$ \\
\hline \multicolumn{4}{|l|}{ SOLANACEAE } \\
\hline Acnistus arborescens (L.) Schltdl. & Fumo bravo & $\mathrm{N}$ & Af, C, Or \\
\hline Cestrum intermedium Sendtn. & Erva de sabiá, pimenta de sabiá* & $\mathrm{N}$ & Af, Or \\
\hline Solanum granulosoleprosum Dunal & Cuvitinga & $\mathrm{N}$ & Af, Or, B \\
\hline Solanum paniculatum L. & Jurubeba & $\mathrm{N}$ & M \\
\hline Solanum pseudoquina A.St.-Hil. & Quina branca & $\mathrm{N}$ & Af, Fl, C, Or, B, T \\
\hline \multicolumn{4}{|l|}{ URTICACEAE } \\
\hline Cecropia spp. & Embaúba & & Af, Fd, Fl, C, Or, M \\
\hline Cecropia glaziovii Snethl. & Embaúva vermelha & $\mathrm{N}$ & Af, Or, M, \\
\hline Cecropia pachystachya Trécul & Embaúva branca & $\mathrm{N}$ & $\mathrm{Fl}$ \\
\hline Coussapoa microcarpa (Schott) Rizzini & Figueira mata pau & $\mathrm{N}$ & Or \\
\hline \multicolumn{4}{|l|}{ VERBENACEAE } \\
\hline Aloysia virgata (Ruiz \& Pav.) Juss. & Repeludo & $\mathrm{N}$ & Af, Fl, B \\
\hline Citharexylum myrianthum Cham. & Jacataiuva & $\mathrm{N}$ & $\mathrm{Fl}, \mathrm{C}, \mathrm{B}$ \\
\hline \multicolumn{4}{|l|}{ VOCHYSIACEAE } \\
\hline Vochysia magnifica Warm. & Guaricica, guarapicica* & $\mathrm{N}$ & C \\
\hline \multicolumn{4}{|l|}{ UNDERTERMINED } \\
\hline Undetermined 1 & Aurêque & & Or \\
\hline Undetermined 2 & Calvi & & $C$ \\
\hline Undetermined 3 & Canela bataieira & & C \\
\hline Undetermined 4 & Canela bosta & & C \\
\hline Undetermined 5 & Canela carne de paca & & $\mathrm{Fl}, \mathrm{C}, \mathrm{Or}$ \\
\hline Undetermined 6 & Canela cascuda & & C \\
\hline Undetermined 7 & Canela coqueiro & & C, Or, T \\
\hline Undetermined 8 & Canela embuia, embuia & & C \\
\hline Undetermined 9 & Canelinha & & Af, C, Or, T \\
\hline Undetermined 10 & Caxeta & & C, $\mathrm{T}$ \\
\hline Undetermined 11 & Ceboleiro & & C \\
\hline Undetermined 12 & Cedro rosa & & C \\
\hline Undetermined 13 & Comocaia & & $\mathrm{Fd}$ \\
\hline Undetermined 14 & Coração de bugre & & $\mathrm{Fl}, \mathrm{C}, \mathrm{T}$ \\
\hline Undetermined 15 & Cuvatã & & C, $\mathrm{T}$ \\
\hline Undetermined 16 & Figueira & & Af, Fl, C, Or, T \\
\hline Undetermined 17 & Figueira preta & & Af, C, Or \\
\hline Undetermined 18 & Figueira verdadeira & & Af, Or \\
\hline Undetermined 19 & Flor de cuitelo & & Or \\
\hline Undetermined 20 & Guaçatunga branca & & C, B \\
\hline Undetermined 21 & Guaçatunga preta & & C, B \\
\hline Undetermined 22 & Guapeba & & $C$ \\
\hline Undetermined 23 & Guaraqui & & $C, \mathrm{~T}$ \\
\hline Undetermined 24 & Imbira caboclo amarela & & Or \\
\hline Undetermined 25 & Ipê & & $\mathrm{C}, \mathrm{M}, \mathrm{T}$ \\
\hline
\end{tabular}


Table 1. Cont.

\begin{tabular}{|c|c|c|c|}
\hline Botanical families/Scientific name & Local name & Origin & Use categories \\
\hline Undetermined 26 & Ipê branco & & C, $\mathrm{T}$ \\
\hline Undetermined 27 & Ipê rosa & & C \\
\hline Undetermined 28 & Jacatirão branco & & $\mathrm{Fl}, \mathrm{C}$ \\
\hline Undetermined 29 & Jacatirão preto & & $\mathrm{Fl}$ \\
\hline Undetermined 30 & Jaracacha & & $\mathrm{Fd}$ \\
\hline Undetermined 31 & Jequitibá & & C \\
\hline Undetermined 32 & Juá & & M \\
\hline Undetermined 33 & Madeira de são joão & & C \\
\hline Undetermined 34 & Maria preta & & C, T, Or \\
\hline Undetermined 35 & Pau de sangue branco & & C \\
\hline Undetermined 36 & Pau de sangue vermelho & & M \\
\hline Undetermined 37 & Pau gambá & & M \\
\hline Undetermined 38 & Pau de sangue, insangueiro & & Or, C \\
\hline Undetermined 39 & Peroba amarela & & $\mathrm{C}, \mathrm{T}$ \\
\hline Undetermined 40 & Peroba escura & & C \\
\hline Undetermined 41 & Peroba graúda & & C \\
\hline Undetermined 42 & Peroba miúda & & C \\
\hline Undetermined 43 & Peroba rosa & & C \\
\hline Undetermined 44 & Vapuana & & Af, $C$ \\
\hline
\end{tabular}

Table 2. Analysis of the influence of gender on the knowledge of local tree species among farmers who are members of Cooperafloresta, the Vale do Ribeira, Brazil, in different use categories. TN: total number of cited species, TA: total average of cited species, NC: number of cited native species, NE: number of cited exotic species, and Ec: number of cited exclusive species. H: men ( $n=23)$, M: women (n = 17). Data are presented as means \pm standard deviations. Different letters in the same row indicate significant differences through Chi-Square* $(\mathrm{p}<0.05)$ and Kruskal-Wallis** $(\mathrm{p}<0.05)$ tests. ${ }^{*}$ denotes sampling sizes insufficient to perform statistical testing.

\begin{tabular}{|c|c|c|c|c|c|c|c|c|c|c|}
\hline \multirow{2}{*}{ Categories } & \multicolumn{2}{|c|}{$\mathrm{TN}^{*}$} & \multicolumn{2}{|c|}{$T A^{* *}$} & \multicolumn{2}{|c|}{ NC* } & \multicolumn{2}{|c|}{$\mathrm{NE}^{\star}$} & \multicolumn{2}{|c|}{ Ec } \\
\hline & $M$ & W & $M$ & W & $M$ & W & $M$ & W & $M$ & $w$ \\
\hline Food & $75^{\mathrm{a}}$ & $68^{a}$ & $17.91 \pm 7.30^{\mathrm{a}}$ & $16.88 \pm 5.87^{a}$ & $40^{a}$ & $35^{a}$ & $35^{\mathrm{a}}$ & $33^{a}$ & $14^{\mathrm{a}}$ & $7^{\mathrm{a}}$ \\
\hline Construction & $74^{\mathrm{a}}$ & $52^{\mathrm{a}}$ & $14.83 \pm 9.31^{b}$ & $7.59 \pm 4.47^{a}$ & $65^{\mathrm{a}}$ & $44^{\mathrm{a}}$ & $9^{\mathrm{a}}$ & $8^{a}$ & $29^{b}$ & $7^{\mathrm{a}}$ \\
\hline Org. matter & $65^{a}$ & $50^{\mathrm{a}}$ & $6.83 \pm 5.37^{\mathrm{a}}$ & $5.59 \pm 3.24^{\mathrm{a}}$ & $53^{a}$ & $39^{a}$ & $12^{\mathrm{a}}$ & $11^{\mathrm{a}}$ & $31^{b}$ & $16^{\mathrm{a}}$ \\
\hline Fuel & $59^{a}$ & $44^{\mathrm{a}}$ & $5.78 \pm 4.04^{\mathrm{a}}$ & $6.18 \pm 3.15^{\mathrm{a}}$ & $53^{a}$ & $38^{\mathrm{a}}$ & $6^{a}$ & $6^{a}$ & $22^{b}$ & $7^{\mathrm{a}}$ \\
\hline Medicine & $47^{\mathrm{a}}$ & $53^{a}$ & $5.22 \pm 3.64^{\mathrm{a}}$ & $8.29 \pm 5.44^{\mathrm{a}}$ & $37^{\mathrm{a}}$ & $41^{\mathrm{a}}$ & $10^{\mathrm{a}}$ & $12^{\mathrm{a}}$ & $10^{\mathrm{a}}$ & $16^{a}$ \\
\hline Animal feed & $44^{\mathrm{a}}$ & $28^{a}$ & $3.39 \pm 3.66^{a}$ & $2.88 \pm 2.55^{\mathrm{a}}$ & $36^{a}$ & $21^{\mathrm{a}}$ & $8^{a}$ & $7^{\mathrm{a}}$ & $31^{b}$ & $15^{\mathrm{a}}$ \\
\hline Technology & $44^{\mathrm{a}}$ & $27^{\mathrm{a}}$ & $4.57 \pm 3.63^{a}$ & $2.53 \pm 2.43^{a}$ & $42^{b}$ & $23^{a}$ & $2^{x}$ & $4^{x}$ & $26^{b}$ & $9^{a}$ \\
\hline Others & $25^{\mathrm{a}}$ & $34^{\mathrm{a}}$ & $2.17 \pm 2.08^{\mathrm{a}}$ & $2.94 \pm 3.17^{\mathrm{a}}$ & $22^{\mathrm{a}}$ & $30^{\mathrm{a}}$ & $3^{x}$ & $4^{\mathrm{x}}$ & $11^{\mathrm{a}}$ & $20^{a}$ \\
\hline Beekeeping & $37^{b}$ & $19^{a}$ & $2.91 \pm 4.28^{\mathrm{a}}$ & $1.53 \pm 2.12^{\mathrm{a}}$ & $29^{b}$ & $12^{\mathrm{a}}$ & $8^{x}$ & $7^{x}$ & $24^{b}$ & $6^{a}$ \\
\hline
\end{tabular}

Table 3. A variance analysis of tree species knowledge by age group of farmers associated with Cooperafloresta, the Vale do Ribeira, Brazil, across different use categories. TN: total number of cited species, TA: total average of cited species, NC: number of cited native species, NE: number of cited exotic species, Ec: total number of cited exclusive species. J: young person (18-24 years, $n=4)$, A: adult (25-59 years, $n=25$ ) and I: elderly (>60 years, $n=11)$. Data are presented as means \pm standard deviations. Different letters in the same row indicate significant differences through Chi-Square* $(\mathrm{p}<0.05)$ and Kruskal-Wallis** $(\mathrm{p}<0.05)$ tests. ${ }^{\mathrm{x}}$ denotes sampling sizes insufficient to perform statistical testing.

\begin{tabular}{|c|c|c|c|c|c|c|c|c|c|c|c|c|c|c|c|}
\hline \multirow{2}{*}{ Categories } & \multicolumn{3}{|c|}{ TN* } & \multicolumn{3}{|c|}{$\mathrm{AvT}^{\star \star \star}$} & \multicolumn{3}{|c|}{$N C^{*}$} & \multicolumn{3}{|c|}{$\mathrm{NE}^{*}$} & \multicolumn{3}{|c|}{ Ex } \\
\hline & Y & A & E & Y & A & E & Y & A & E & Y & A & E & Y & A & E \\
\hline Food & $42^{a}$ & $69^{b}$ & $66^{\mathrm{b}}$ & $20.25 \pm 12.84^{a}$ & $16.72 \pm 5.13^{a}$ & $18.18 \pm 7.80^{\mathrm{a}}$ & $13^{\mathrm{a}}$ & $36^{\mathrm{b}}$ & $33^{b}$ & $29^{a}$ & $33^{a}$ & $33^{a}$ & $1^{\mathrm{a}}$ & $14^{\mathrm{b}}$ & $9^{b}$ \\
\hline Construction & $16^{\mathrm{a}}$ & $71^{\mathrm{b}}$ & $63^{b}$ & $5.75 \pm 5.44^{\mathrm{a}}$ & $10.96 \pm 7.24^{\mathrm{a}}$ & $15.73 \pm 10.29^{a}$ & $12^{\mathrm{a}}$ & $61^{b}$ & $56^{\mathrm{b}}$ & $4^{\mathrm{a}}$ & $10^{\mathrm{a}}$ & $7^{\mathrm{a}}$ & $1^{\mathrm{a}}$ & $17^{\mathrm{b}}$ & $8^{b}$ \\
\hline Org. matter & $17^{\mathrm{a}}$ & $71^{c}$ & $33^{b}$ & $5.75 \pm 3.10^{\mathrm{a}}$ & $7.04 \pm 4.98^{\mathrm{a}}$ & $4.82 \pm 3.95^{\mathrm{a}}$ & $13^{\mathrm{a}}$ & $56^{c}$ & $30^{b}$ & $4^{a}$ & $15^{b}$ & $3^{a}$ & $1^{\mathrm{a}}$ & $38^{b}$ & $8^{\mathrm{a}}$ \\
\hline Fuel & $9^{\mathrm{a}}$ & $58^{c}$ & $32^{\mathrm{b}}$ & $2.25 \pm 1.89^{\mathrm{a}}$ & $6.84 \pm 3.53^{b}$ & $5.27 \pm 3.61^{\mathrm{ab}}$ & $8^{\mathrm{a}}$ & $50^{c}$ & $30^{\mathrm{b}}$ & $1^{\mathrm{x}}$ & $8^{x}$ & $2^{\mathrm{x}}$ & $1^{\mathrm{a}}$ & $31^{\mathrm{b}}$ & $6^{\mathrm{a}}$ \\
\hline Medicine & $4^{\mathrm{a}}$ & $56^{c}$ & $32^{b}$ & $1.00 \pm 1.15^{\mathrm{a}}$ & $7.80 \pm 4.86^{b}$ & $5.64 \pm 3.41^{b}$ & $4^{a}$ & $44^{c}$ & $25^{b}$ & $0^{\mathrm{a}}$ & $12^{\mathrm{b}}$ & $7^{b}$ & $0^{\mathrm{a}}$ & $31^{c}$ & $7^{b}$ \\
\hline Animal feed & $0^{\mathrm{a}}$ & $43^{b}$ & $37^{\mathrm{b}}$ & $0^{\mathrm{a}}$ & $3.8 \pm 2.84^{b}$ & $4.55 \pm 3.83^{b}$ & $0^{\mathrm{a}}$ & $33^{b}$ & $31^{\mathrm{b}}$ & $0^{\mathrm{a}}$ & $10^{\mathrm{b}}$ & $6^{\mathrm{b}}$ & $0^{\mathrm{a}}$ & $22^{\mathrm{b}}$ & $16^{\mathrm{b}}$ \\
\hline Technology & $1^{\mathrm{a}}$ & $44^{\mathrm{b}}$ & $32^{\mathrm{b}}$ & $0.25 \pm 0.50^{\mathrm{a}}$ & $3.28 \pm 2.79^{b}$ & $5.91 \pm 3.62^{c}$ & $1^{\mathrm{a}}$ & $39^{b}$ & $30^{\mathrm{b}}$ & $0^{\mathrm{x}}$ & $5^{x}$ & $2^{\mathrm{x}}$ & $0^{\mathrm{a}}$ & $21^{c}$ & $9^{\mathrm{b}}$ \\
\hline Others & $5^{a}$ & $29^{b}$ & $28^{\mathrm{b}}$ & $1.25 \pm 0.50^{\mathrm{a}}$ & $2.20 \pm 2.16^{a}$ & $3.64 \pm 3.56^{a}$ & $3^{a}$ & $25^{\mathrm{b}}$ & $26^{\mathrm{b}}$ & $2^{\mathrm{x}}$ & $4^{x}$ & $2^{x}$ & $0^{\mathrm{a}}$ & $15^{\mathrm{b}}$ & $14^{\mathrm{b}}$ \\
\hline Beekeeping & $6^{a}$ & $32^{b}$ & $21^{b}$ & $1.50 \pm 3.00^{\mathrm{a}}$ & $2.52 \pm 4.00^{\mathrm{a}}$ & $2.18 \pm 2.82^{\mathrm{a}}$ & $4^{a}$ & $24^{\mathrm{b}}$ & $16^{\mathrm{b}}$ & $2^{x}$ & $8^{a}$ & $5^{a}$ & $0^{\mathrm{a}}$ & $19^{b}$ & $11^{\mathrm{b}}$ \\
\hline
\end{tabular}


Considering the plants reported exclusively among age groups, the number of species cited by adults (29 sp.) was higher than that cited by youngsters ( 2 sp., $X^{2}=23.51$, $\mathrm{p}<$ $0.0001)$ and the elderly ( 9 sp., $X^{2}=10.52, p=0.0021$ ) and was significantly different regarding the use categories: organic matter (adult $\times$ youngster, $X^{2}=35.10, \mathrm{p}<0.0001$; adult $\times$ elderly, $X^{2}=19.56, \mathrm{p}<0.0001$ ), fuel (adult $\times$ youngster, $X^{2}$ $=28.12, \mathrm{p}<0.0001$; adult $\times$ elderly, $\left.X^{2}=16.89, \mathrm{p}<0.0001\right)$, medicine (adult $\times$ youngster, $X^{2}=31, \mathrm{p}<0.0001$; adult $\times$ elderly, $X^{2}=15.15, \mathrm{p}=0.0002$ ) and technology (adult $\times$ youngster, $X^{2}=21, \mathrm{p}<0.0001$; adult $\times$ elderly, $X^{2}=4.8, \mathrm{p}=$ 0.0446 ). The species mentioned only by young people were in the food and construction categories, whereas the plants known exclusively by the elderly were in the animal feed (3 sp.), fuel (1 sp.), organic matter (2 sp.), medicine (1 sp.), technology (1 sp.) and other categories (4 sp.).

\section{Discussion}

\section{Knowledge in relation to gender}

Homogeneous knowledge between the genders has been reported by other studies (Sop et al. 2012; Brandt et al. 2013) and may be related to the knowledge is transmitted (ReyesGarcía et al. 2009). In this case, the collective work helped agroforestry management to become a rich opportunity for learning and sharing experiences among all the participants (Steenbock et al. 2013). Collective work is often used to manage tasks such as opening new areas and planting that require extra manpower. However, the training efforts are aimed at integrating technical and scientific knowledge with local knowledge to develop improved production strategies, commercialization and product certification. In this sense, Reyes-García et al. (2009) highlighted the importance of social relations in the construction of knowledge, in which the status of an individual in a network of information may explain the levels of the knowledge that individual acquires.

However, the fact that some species were exclusively mentioned by each gender indicates specialized knowledge in certain use categories because women and men have distinct jobs and social interactions (Howard 2003; CamouGuerrero et al. 2008; Reyes-Garcia et al. 2010). Previous studies have found that women have greater knowledge of native species for food and medicinal use (Voeks \& Leony 2004; Camou-Guerrero et al. 2008; Guimbo et al. 2011), whereas men possess more knowledge about native tree species used in construction and technology (EstradaCastillón et al. 2014; Lyon \& Hardesty 2014; Ramos et al. 2015).

This knowledge divergence may relate to the farmers' relationships with biodiversity (Zuchiwschi et al. 2010; Albuquerque et al. 2011). In the past, attempts to diversify the local economy through extensive cattle farming and exploitation of palm and timber resources may have put men in closer contact with the local flora. The difficult access and long distances to work locations from residences meant that these activities were mainly conducted by men; women had limited time to stay in these places. Moreover, the work of managing these plant resources often involved physical issues. This disparity may explain why men were able to mention a greater number of exclusive species used as timber.

Currently, men still go travel to forested areas more often and work there longer than women because of the distance between these locations and people's homes. Despite conducting the same type of farming activity, the responsibilities of each gender were somewhat different. Sometimes, women and men work together to determine planting times, the planted varieties, the amount of stored seeds and whether they were bought or traded, which are the main factors in preserving biodiversity "in situ". In this case, men leave in the morning or late in the afternoon to perform pruning, weeding or harvesting in the more distant agroforestry fields, while women travel to these locations less frequently. Instead, the women care for backyard areas and agroforest orchards located close to their homes as well as assume the primary family caregiver roles such as meal preparation and the treatment of diseases. However, there are a few families in which only women are members and bear the primary responsibility for agroforestry management-in addition to taking care of the family. This finding corroborates with worldwide growth in the number of women who have assumed the householder role in rural areas (1/3 of homes around the world, Howard \& Nabanoga 2007) due to the migration of men to the cities.

\section{Knowledge in relation to age}

Several authors have reported that individuals in their respective studies became more knowledgeable as their age increased (Mathez-Stiefel et al. 2012; Sop et al. 2012). One of the reasons why older people have accumulated more knowledge than younger people may be related to the experiences these individuals have during their lives. In this sense, higher average species mentions and higher exclusive species mentions by the elderly in the technology category may be related to the experiences and opportunities that occurred during each individual's life (Phillips \& Gentry 1993). Canoes, for example, were widely used by farmers in the past as a means of transportation. However, the construction of the Capivari-Cachoeira dam system reduced the flow of the Turvo River, hindering river navigation in the region. In addition to canoes, many farmers made household items such as sieves, spoons, mortars and pestles from native flora. However, many of these items have been replaced by manufactured versions or are no longer used, reducing the need for technological uses of the native flora by the younger population.

The greatest richness of useful species mentioned by 
the 25-59 age group involved the organic matter, fuel and medicine categories. This result may indicate that adults have greater contact with the plant resources used for these purposes. Estrada-Castillón et al. (2014) found an accumulated knowledge regarding the number of citations of useful species for the same age group in a population in semi-arid Mexico. People in this age group may be more active in agroforestry management and recognize the importance of vegetation cover; therefore, they experiment with several species. However, a greater knowledge of species for use as fuel may be associated with lower costs: people more often use wood than gas stoves to prepare food. Adults also cited a greater number of exotic species in the construction category. This result may be related to current environmental legislation that encourages the abandonment and replacement of native species by exotic ones (Oliveira Jr et al. 2013).

Moreover, learning takes time, which may be one reason why older people tend to have accumulated more knowledge than young people. Young people's repertoire of information regarding the local flora may increase as they have more opportunities to learn about them. However, some studies show that age cannot be a differentiating factor in the variation of knowledge because young people attend the houses of elders to learn their teachings (Martins et al. 2014). In this regard, Cooperafloresta has a training programme on agroforestry for children and adolescents, in which the members' children meet regularly with educators and other farmers to learn about agroecology principles and agroforestry practices. Another objective of this training is to minimize migration by young people by providing them with tools that allow them to be engaged with the association.

The small number of young people interviewed shows that they have mostly moved to urban centres in search of better employment opportunities and access to education. Farmers in this study supported the access of their children to formal education, but - unlike those observed by Legesse et al. (2013), the parents also encourage their children to undertake activities related to agroforestry to make a higher quality of life possible. There are many cases of children who studied abroad so that they could bring back their newly acquired expertise to their place of origin after graduation. However, when the young people do not return, migration also affects knowledge preservation by causing a break in the transmission of information that can affect the knowledge of the use of plant resources in the long term.

It is important to remember that this study made a deliberate selection of participants who have greater knowledge of local resources. This limits the interpretation of the results because they represent this particular group's knowledge rather than the knowledge of the entire community. Unexpectedly, the results showed no differences in the total knowledge of mentioned species between genders, but when considering the species reported exclusively by each group, the men mentioned significantly higher numbers of species, which may indicate the existence of specialized knowledge in this group for certain categories. As expected, age proved to be a differentiating factor: young people cited fewer species compared with adults and the elderly, and the fact that the experiences occur throughout each individual's lifetime may be related to older people's greater accumulation of knowledge.

The ethnoknowledge in agroforestry systems differs from that in groups with more traditional relationships with the forest. This difference occurs because agroforestry systems-especially those with an agroecological base, as in Cooperafloresta -include components such as feminism and the empowerment of young people, which can introduce differences in the pattern of knowledge between gender and age. Another interesting point is that young people were traditionally included in the family dynamics, working as assistants to their parents, fostering strong knowledge transmission. Among young agroforestry farmers we find that young protagonists act with more autonomy when compared to historical times and, in addition to family dynamics, they obtain knowledge from other sources such as joint efforts, workshops and interactions with technical experts (not necessarily only through formal education), all of which are much more prevalent today.

\section{Acknowledgements}

The authors extend their appreciation and gratitude to the farmers who contribute to the survey; to Carlos Carriel and Rodrigo Ozelame, who facilitated the initial dialogue; to the support by Fundação Florestal, especially Wagner Portilho and Airton Vieira; to the Coordenação de Aperfeiçoamento de Pessoal de Nível Superior (CAPES) for the scholarship granted to the first author; and finally, to Sonia Aragaki Filomena Dias, Geraldo Franco, João Baitello and Osny de Aguiaro for their efforts in botanical identification.

\section{References}

Adams C, Munari LC, Vliet N, et al. 2013. Diversifying Incomes and losing landscape complexity in quilombola shifting cultivation communities of the Atlantic Rainforest (Brazil). Human Ecology 41: 119-137.

Albuquerque UP. 1997. Etnobotânica: uma aproximação teórica e epistemológica. Revista Brasileira Farmácia 78: 60-64.

Albuquerque UP, Ramos MA, Lucena RFP, Alencar NL. 2014. Methods and techniques used to collect ethnobiological data. In: Albuquerque UP, Cunha LVF, Alves RRN. (eds.) Methods and techniques in ethnobiology and ethnoecology. New York, Springer. p. 15-38.

Albuquerque UP, Soldati GT, Siebera SS, Ramos MA, SÁ JC, Souza LC. 2011. The use of plants in the medical system of the Fulni-ô people (NE Brazil): A perspective on age and gender. Journal of Ethnopharmacology 133: 866-873.

Altieri MA, Nicholls CI. 2012. Diálogo de saberes: agroecólogos e agricultores por uma agricultura verdadeiramente sustentável. In: Altieri MA. (ed.) Agroecologia: bases científicas para uma agricultura sustentável. São Paulo/Rio de Janeiro, Expressão Popular (AS-PTA). p. 159-182.

Alves HPF. 2004. Análise dos fatores associados às mudanças na cobertura da terra no Vale do Ribeira através da integração de dados censitários e de sensoriamento remoto. Campinas, Núcleo de Estudos de População/ UNICAMP. 


\section{Do gender and age influence agroforestry farmers' knowledge of tree species uses in an area of the Atlantic Forest, Brazil?}

Andrade AM, Tatto N. 2013. Inventário cultural de quilombos do Vale do Ribeira. São Paulo, Instituto Socioambiental.

Araújo TAS, Almeida ALA, Melo JG, et al. 2012. A new technique for testing distribution of knowledge and to estimate sampling sufficiency in ethnobiology studies. Journal of Ethnobiology and Ethnomedicine 8: 11.

Assogbadjo AE, Kakaï RG, Vodouhê FG, Djagoun CAMS, Codjia JTC, Sinsin B. 2012. Biodiversity and socioeconomic factors supporting farmers' choice of wild edible trees in the agroforestry systems of Benin (West Africa). Forest Policy and Economics 14: 41-49.

Avilez W, Medeiros P, Albuquerque UP. 2016. Effect of gender on the knowledge of medicinal plants: systematic review and meta-analysis. Evidence-Based Complementary and Alternative Medicine. Doi: http:// dx.doi.org/10.1155/2016/6592363

Ayres M, Ayres Jr M. 2007. BioEstat versão 5.0—Aplicações estatísticas nas áreas das ciências bio-médicas. 5th. edn. Belém, Mamirauá.

Begossi A. 1996. Use of ecological methods in ethnobotany: diversity índices. Economic Botany 50: 280-289.

Brandt R, Mathez-Stiefel S, Lachmuth S, Hensen I, Rist S. 2013. Knowledge and valuation of Andean agroforestry species: the role of gender, age, and migration among members of a rural community in Bolivia. Journal of Ethnobiology and Ethnomedicine 9: 83.

Brasil 2000. Lei n. 9.985, de 18 de julho de 2000. Regulamenta o art. 225 , $§ 1^{\circ}$, incisos I, II, III e VII da Constituição Federal, institui o Sistema Nacional de Unidades de Conservação da Natureza e dá outras providências. Brasília, Diário Oficial [da] República Federativa do Brasil.

Camou-Guerrero A, Reyes-García V, Martínez-Ramos M, Casas A. 2008. Knowledge and use value of plant species in a Rarámuri community: a gender perspective for conservation. Human Ecology 36: 259-272.

Case RJ, Guido FP, Soejarto DD. 2005. Factors in maintaining indigenous knowledge among ethinic communities of Manus Island. Economic Botany 59: 356-365.

Ceuterick M, Vandebroek I, Torrya B, Pieroni A. 2008. Cross-cultural adaptation in urban ethnobotany: the Colombian folk pharmacopoeia. Journal of Ethnopharmacology 120: 342-359.

Collwell RK. 2013. EstimateS: statistical estimation of species richness and shared species from samples. Version 9.0. http://viceroy.eeb. uconn.edu/EstimateS

Collwell RK, Coddington JA. 1994. Estimating terrestrial biodiversity through extrapolation. Philosophical Transactions of the Royal Society of London 345: 101-118.

Cunha LVFC, Albuquerque UP. 2006. Quantitative ethnobotany in an Atlantic Forest fragment of northeastern Brazil - implications to conservation. Environmental Monitoring and Assessment 114: 1-25.

Devide AC, Castro C, Lima Jr EO, et al. 2013. 'Mutirão Agroflorestal': herramienta de red de agroforestería del Vale do Paraíba, Brasil. In: Altieri MA, Sarandon S, Morales CF, Funes F, Siura S. (eds.) Congresso Latinoamericano de Agroecología: artículos completos. Lima, Sociedad Científica Latinoamericana de Agroecología (SOCLA), Universidad Nacional Agraria La Molina e IFOAM América Latina.

Diegues ACS, Arruda RSV. 2001. Saberes tradicionais e biodiversidade no Brasil. Brasília, MMA série Biodiversidade.

Estrada-Castillón E, Garza-López M, Villarreal-Quintanilla JÁ, et al. 2014. Ethnobotany in Rayones, Nuevo León, México. Journal of Ethnobiology and Ethnomedicine 10: 62.

Gandolfo ES, Hanazaki N. 2011. Ethnobotany and urbanization: knowledge and use of restinga plants by the native community of Distrito do Campeche (Florianópolis, Santa Catarina, Brazil). Acta Botanica Brasilica 25: 168-177.

García-Barrios L, Ong CK. 2004. Ecological interactions, management lessons and design tools in tropical agroforestry systems. Agroforestry Systems 61: 221-236.

Gomes EPC, Sugiyama M, Adams C, Prado HM, Oliveira Jr CJF. 2013. A sucessão florestal em roças em pousio: a natureza está fora da lei? Scientia Forestalis 41: 343-352.

Guimbo ID, Muller J, Larwanou M. 2011. Ethnobotanical knowledge of men, women and children in rural Niger: a mixed methods approach. Ethnobotany Research \& Applications 9: 235-242.
Hanazaki N, Tamashiro JY, Leitão Filho HF, Begossi A. 2000. Diversity of plant uses in two caiçara communities from the Atlantic Forest coast, Brazil. Biodiversity and Conservation Journal 9: 5 .

Howard P, Nabanoga G. 2007. Are there customary rights to plants? An inquiry among the Baganda (Uganda), with special attention to gender. World Development 35: 1542-1563.

Howard P. 2003. Women and the plant world: An exploration. In Howard P. (ed.) Women and plants: Gender relations in biodiversity management and conservation. London/New York, Zed Press and Palgrave-Macmillan. p. 1-48.

IBGE - Instituto Brasileiro de Geografia e Estatística. 2010. Censo Demográfico 2010. http://www.ibge.gov.br/.

IBGE Cidades - Instituto Brasileiro de Geografia e Estatística. 2016. Síntese das Informações. http://cidades.ibge.gov.br/xtras/perfil.php?lang=\& codmun $=350540 \&$ search=sao-paulo $\mid$ barra-do-turvo

Ivanauskas NM, Miashike RL, Godoy JRL, et al. 2012. A vegetação do Parque Estadual Turístico do Alto Ribeira (PETAR), São Paulo, Brasil. Biota Neotropica 12: 147-177.

Kang BT, Akinnifesi F. K. 2000. Agroforestry as alternative land-use production systems for the tropics. Natural Resources Forum 24: 137-151.

Legesse A, Teferi B, Baudouin A. 2013. Indigenous agroforestry knowledge transmission and young people's participation in agroforestry practices: The case of Wonago Woreda, Gedeo Zone, Southern Ethiopia. Acta Geographica-Trondheim Serie A, 26: 31.

Lepsh IF. 2002. Formação e conservação dos solos. São Paulo, Oficina de Textos.

Lepsh IF, Saraiva IR, Donzeli PL. et al. 1990. Macrozoneamento das terras da região do rio Ribeira de Iguape, SP. Boletim Científico/Instituto Agronômico de Campinas 19: 1-181.

Lunelli NP, Ramos SF, Oliviera Jr CJF. 2013. Agroflorestas e externalidades. Revista Verde de Agroecologia e Desenvolvimento Sustentável 8: 163-170.

Lyon LM, Hardesty LH. 2014. Quantifying medicinal plant knowledge among non-specialist antanosy villagers in Southern Madagascar. Economic Botany 66: 1-11.

Martins RC, Filgueiras TS, Albuquerque UP. 2014. Use and diversity of palm (Arecaceae) resources in central western Brazil. The Scientific World Journal. http://dx.doi.org/10.1155/2014/942043

Mathez-Stiefel SL, Vandebroek I. 2012. Distribution and transmission of medicinal plant knowledge in the Andean Highlands: a case study from Peru and Bolivia. Evidence-Based Complementary and Alternative Medicine doi: 10.1155/2012/959285

Neves WA, Murrieta RSS, Adams C, Ribeiro Filho AA, Pedroso Jr NN. 2012. Coivara: cultivo itinerante na floresta tropical. Ciência Hoje 50: 26-30.

Oliveira Jr CJF, Cabreira PP. 2012. Sistemas Agroflorestais: potencial econômico da biodiversidade vegetal a partir do conhecimento tradicional ou local. Revista Verde de Agroecologia e Desenvolvimento Sustentável 7: 212-224.

Oliveira Jr CJF, Gonçalves FS, Couto F, Matajs L. 2013. Potencial das espécies nativas na produção de plantas ornamentais e paisagismo agroecológico. Revista Brasileira de Agroecologia 8: 190-200.

Pfeiffer JM, Butz RJ. 2005. Assessing cultural and ecological variation in ethnobiological research: the importance of gender. Journal of Ethnobiology 25: 240-278.

Phillips O, Gentry AH. 1993. The useful plants of Tambopata, Peru: II. Additional hypothesis testing in quantitative ethnobotany. Economic Botany 47: 33-43.

Quinlan MB, Quinlan RJ. 2007. Modernization and medicinal plant knowledge in a Caribbean horticultural village. Medical Anthropology Quarterly 21: 169-192.

Ramos MA, Lucena RFP, Albuquerque UP. 2015. What drives the knowledge and local uses of timber resources in human-altered landscapes in the semiarid region of northeast Brazil? International Journal of Sustainable Development \& World Ecology 22: 5.

Ramos MA, Medeiros PM, Albuquerque UP. 2014. Methods and techniques applied to ethnobotanical studies of timber resources. In: Albuquerque UP, Cunha LVF, Alves RRN. (eds.) Methods and techniques in ethnobiology and ethnoecology. New York, Springer. p. 349-366 
Reyes-García V, Broesch J, Calvet-Mir L, et al. 2009. Cultural transmission of ethnobotanical knowledge and skills: an empirical analysis from an Amerindian society. Evolution and Human Behaviour 30: 274-285.

Reyes-García V, Valdez V, Huanca T, Wilkie WLD. 2005. Knowledge and consumption of wild plants: a comparative study in two Tsimane' villages in the Bolivian Amazon. Ethnobothany Research \& Applications 3: 201-207.

Reyes-García V, Vila S, Ceituno-Mata LA, et al. 2010. Gendered homegardens: a study in three mountain areas of the Iberian Peninsula. Economic Botany 64: 235-247.

Scherr SJ. 1991. On-farm research: the challenges os agroforestry. Agroforestry Systems 15: 95-110.

Setzer J. 1966. Atlas climático e ecológico do estado de São Paulo. São Paulo, Comissão Interestadual da Bacia Paraná-Uruguai.

Shiva V. 2002. Monoculturas da Mente: perspectivas da biodiversidade e da biotecnologia. São Paulo, Gaia.

SIT - Sistema de Informações Territoriais. 2013. http://sit.mda.gov.br

Sop TK, Oldeland J, Bognounou F, Schmiedel U, Thiombiano A. 2012.
Ethnobotanical knowledge and valuation of woody plants species: a comparative analysis of three ethnic groups from the sub-Sahel of Burkina Faso. Environment, Development and Sustainability 14: 627-649.

Steenbock W, Seoane CE, Froufe LCM. 2013. Agrofloresta, ecologia e sociedade. Curitiba, Kairós.

Tongco MDC. 2007. Purposive sampling as a tool for informant selection. Ethnobotany Research \& Applications 5: 147-158.

Voeks RA, Leony A. 2004. Forgetting the forest: assessing medicinal plant erosion in eastern Brazil. Economic Botany 58: S294-S306.

Williams VL, Witkowski ETF, Balkwill K. 2007. The use of incidence-based species richness estimators, species accumulation curves and similarity measures to appraise ethnobotanical inventories from South Africa. Biodiversity Conservation 16: 2495-2513.

Zuchiwschi E, Fantini AC, Peroni N. 2010. Limitações ao uso de espécies florestais nativas pode contribuir com a erosão do conhecimento ecológico tradicional e local de agricultores familiares. Acta Botanica Brasilica 24: 270-282. 